\title{
ON DESCRIPTION OF THE DIRECT NUCLEON DECAY OF GIANT RESONANCES
}

\author{
G.A.Chekomazov, S.E.Muraviev, M.H.Urin \\ Moscow Engineering Physics Institute, 115409, Moscow, Russia
}

1. The direct nucleon decay of giant resonances (GR) in intermediate and heavy mass nuclei is now the subject of intensive experimental and theoretical studies. At the moment there is a body of experimental data on the GR nucleon decay to certain (singlehole) states of a product nucleus. These data are: (i) the partial proton escape widths of isobaric analog resonance (IAR) (see, e. g., [ [ ] and refs. therein); (ii) the partial cross sections of the photonucleon reactions accompanied by excitation of the giant dipole resonance (GDR) (see, e.g., [3]); (iii) the rather contradictory data on the partial neutron escape widths of the isoscalar monopole giant resonance (GMR) in ${ }^{208} \mathrm{~Pb}$ [4, 5]; (iv) the branching ratios for the direct neutron decay of the GDR and region above in ${ }^{208} \mathrm{~Pb}$ [6]; (v) the partial proton escape widths of the Gamow-Teller resonance (GTR) in ${ }^{208} \mathrm{Bi}$ [7]. The appearance of new experimental data on the direct nucleon decay of various GR is expected in the near future (see, e.g., these Proceedings).

The theoretical description of the direct nucleon decay of GR is a serious test for models of nuclear structure and nuclear reactions, because single-particle, collective, and manyparticle aspects of nuclear motion are combined in this phenomenon. Modern theoretical approaches to the mentioned description are based on the random phase approximation with consideration for the single-particle continuum (on the continuum-RPA) [2,4,8-17]. In these refs. the energies, strengths, and partial escape widths of collective states of the particle-hole ( $\mathrm{p}-\mathrm{h})$ type (these states are called below the doorway states (dws), or the gross-structure components of the considered GR) have been calculated on the basis of different versions of the continuum-RPA with the use of different forms of the nuclear mean field and p-h interaction. Attempts to consider the GR nucleon decay together with explicit consideration for the coupling of the dws to $2 \mathrm{p}-2 \mathrm{~h}$ configurations have been undertaken in [11, 14].

In the present talk the somewhat extended version of the approach developed in [15, 17] is briefly outlined. Applications of the approach to description of the direct nucleon decay of a number of GR in the ${ }^{208} \mathrm{~Pb}$ parent nucleus and a comparison of calculation results with relevant experimental data are given.

2. The main features of the approach are the following. (i) As input data for the RPA calculations we use the phenomenological mean field of the Woods-Saxon type and the phenomenological p-h interaction. This latter is chosen in different forms to clarify the dependence of the calculation results on the choice of the p-h interaction. For the description of IAR and GTR we realize the partial selfconsistency (see, e. g., [2]), which is a result of the approximate isospin-symmetry of the nuclear Hamiltonian. (ii) We use the exact version of the continuum-RPA for calculating the amplitudes of those partial $\left(e, N_{c}\right)$ - and inclusive reactions, which are studied experimentally $\left(e=N_{c}, \gamma, \ldots\right.$ means the entrance reaction-channel: nucleon, radiative, ...; $c$ is the set of quantum numbers for the nucleon decay-channel). (iii) In the case of GR with not-too-large excitation energy it is possible to use the Breit-Wigner parametrization of the reaction amplitudes calculated within the continuum-RPA for evaluating the dws parameters: the excitation energy, the entrance width (or corresponding strength), the partial escape widths. (iv) We 
consider the doorway-state coupling to many-particle configurations phenomenologically on the average over the energy using a reasonable statistical assumption and the fact that there are no common channels for the nucleon decay of the dws and many-particle states [15. This consideration allows us to describe the energy-averaged reaction-amplitudes by means of an independent spreading (and energy shift) of each dws resonance in the RPA reaction-amplitudes. (v) The adequate consideration of the penetrability of the potential barrier for the escaping nucleon requires the use of (a) the experimental energies of the nucleon decay-channels instead of calculated ones [16]; (b) the penetrability averaged over the GR region. (vi) The possibility to describe the direct nucleon decay of GR in terms of the escape widths depends on the structure of the GR strength function (or of the inclusive reaction cross-section) calculated within the continuum-RPA. (a) When only one dws corresponds to the GR, the energy-averaged reaction cross-sections in the GR region look like a Breit-Wigner resonance. In this case the reaction cross-sections can be described in terms of the partial nucleon escape widths $\Gamma_{c}$ (or of the widths $\left\langle\Gamma_{c}\right\rangle$ averaged over the GR region), which are coincident with those calculated within the continuumRPA and are independent of both the dws coupling to many-particle configurations and the entrance channel [15, 17]. For this reason the entrance channel can be chosen for convenience of calculations. We usually use a nucleon channel as entrance one [15, 16]. (b) In the case of some essential gross-structure it is necessary to calculate the energyaveraged cross-sections of the $\left(e N_{c}\right)$ - reactions $\sigma_{e N_{c}}$ (or the branching ratios $b_{c}^{(e)}=\sigma_{e N_{c}} / \sigma_{e}$, where $\sigma_{e}$ is the energy-averaged inclusive reaction cross-section) and to compare them with experimental data directly. Only in the case, when the root-mean-squared energy dispersion of the dws is less than the spreading width of the dws $\Gamma^{\downarrow}$, the calculated cross section $\sigma_{e N_{c}}$ can be approximately represented in the Breit-Wigner form, and the branching ratios are equal to $b_{c}^{(e)}=\Gamma_{c}^{(e)} / \Gamma^{\downarrow}$, where $\Gamma_{c}^{(e)}$ are effective partial widths of the GR. These widths are determined by the amplitudes of the entrance and escape widths of the dws and, therefore, are dependent on the entrance channel [17].

3. To illustrate the abilities of the approach outlined briefly above let us turn to description of the direct nucleon decay for a number of GR in the ${ }^{208} \mathrm{~Pb}$ parent nucleus. The parametrization and parameters of the phenomenological mean field used in the calculations are given in detail in [15]. The choice of the phenomenological p-h interaction is given below for each considered GR.

Isobaric analog resonance. The theoretical description of the direct proton decay of the IAR is closely related to the approximate conservation of the isospin symmetry in nuclei (see, e. g., [2, 18] and refs. therein). If this symmetry were exact, all nucleon decays of the IAR would be forbidden. Therefore, these decays are determined by the mechanism and strength of the isospin-symmetry violation in nuclei. To take the violation into account most correctly within the shell-model, the isovector part of the nuclear mean field (the symmetry potential) should be consistent with the neutron-excess density via the isovector part of the $\mathrm{p}$-h interaction $F_{\tau}\left(x, x^{\prime}\right)=\boldsymbol{\pi} \boldsymbol{\tau}^{\prime} F_{\tau}\left(\mathbf{r}, \mathbf{r}^{\prime}\right)$, where $x$ is the set of nucleon coordinates including spin and isospin ones (see, e. g., [2]). In calculations we use the Landau-Migdal and separable interactions:

$F_{\tau}\left(\mathbf{r}, \mathbf{r}^{\prime}\right)=F_{\tau} \delta\left(\mathbf{r}-\mathbf{r}^{\prime}\right)$

$F_{\tau}\left(\mathbf{r}, \mathbf{r}^{\prime}\right)=K_{\tau} f_{w s}(r) f_{w s}\left(r^{\prime}\right)$, 
where $f_{w s}(r)$ is the Woods-Saxon function. The strength parameters $F_{\tau}=f^{\prime} \cdot 300$ $\mathrm{MeV} \cdot \mathrm{fm}^{3}, f^{\prime}=0.95$, and $K_{\tau}=0.16 \mathrm{MeV}$ are found by using the mentioned selfconsistency condition and the phenomenological expressions for the symmetry potential and the neutron-excess density. Because the Fermi strength function calculated with the use of interactions (1) has no gross-structure in the IAR region, we calculate the S-matrix of the proton-nucleus scattering and evaluate the partial proton widths of the IAR in the ${ }^{208} \mathrm{~B} i$ by means of the Breit-Wigner parametrization of the S-matrix using the experimental energies of the decay channels. The calculated widths are given in Table 1 together with experimental widths deduced from both the resonance reactions (see refs. in 18]) and the direct reactions [7]. Because the total width of the IAR is small, there is no need to use the penetrability averaged over the resonance. As expected, the calculated widths $\Gamma_{c}$ are dependent on the choice of the $\mathrm{p}-\mathrm{h}$ interaction only slightly, because these widths are mainly determined by the mean Coulomb field [2, 18].

\section{Table 1}

Proton escape widths (in keV) of IAR and GTR in ${ }^{208} \mathrm{Bi}$

\begin{tabular}{|c|c|c|c|c|c|c|c|c|}
\hline \multirow{3}{*}{$\begin{array}{c}\text { final } \\
\text { state } \\
\text { of }{ }^{207} \mathrm{~Pb}\end{array}$} & \multicolumn{4}{|c|}{ IAR } & \multicolumn{4}{|c|}{ GTR } \\
\hline & \multicolumn{2}{|c|}{$\Gamma_{c}(\exp )$} & \multicolumn{2}{|c|}{$\Gamma_{c}($ calc $)$} & \multirow{2}{*}{$\begin{array}{c}\Gamma_{c}(\exp ) \\
\text { ]7 }\end{array}$} & \multirow{2}{*}{$\begin{array}{c}\Gamma_{c}(\text { calc }) \\
15\end{array}$} & \multirow{2}{*}{\multicolumn{2}{|c|}{$\begin{array}{cc}\left\langle\Gamma_{c}\right\rangle & \text { (calc) } \\
\text { int. } & \text { int. } \\
(2 \mathrm{a}) & (2 \mathrm{~b}) \\
\end{array}$}} \\
\hline & 18 & (7) & $\begin{array}{l}\text { int. } \\
\text { (1a) }\end{array}$ & $\begin{array}{l}\text { int. } \\
\text { (1b) }\end{array}$ & & & & \\
\hline $1 / 2^{-}$ & $51.6 \pm 1.7$ & $51.4 \pm 5.6$ & 61 & 63 & $48.9 \pm 9.3$ & 33 & 43 & 173 \\
\hline $5 / 2^{-}$ & $24 \pm 4$ & incl.in $p_{3 / 2}$ & 27 & 31 & incl.in $p_{3 / 2}$ & 18 & 48 & 164 \\
\hline $3 / 2^{-}$ & $54 \pm 7$ & $79.4 \pm 9.4$ & 79 & 86 & $84.9 \pm 13.1$ & 21 & 35 & 218 \\
\hline $13 / 2^{+}$ & & & 0.18 & 0.17 & $6.9 \pm 7.7$ & 0.04 & 0.78 & 5.2 \\
\hline $7 / 2^{-}$ & $5.0 \pm 0.5$ & $3.5 \pm 1.6$ & 8.5 & 8.7 & $13.1 \pm 6.2$ & 0.26 & 8.9 & 78 \\
\hline$\Gamma_{t o t}^{\uparrow}$ & 134.6 & 134.3 & 176 & 189 & 153.8 & 72 & 136 & 638 \\
\hline
\end{tabular}

Gamow-Teller resonance. The partial proton escape widths $\Gamma_{c}$ of the GTR in ${ }^{208} B i$ have been calculated in [15] and [16] with the use of the Landau-Migdal p-h interaction (respectively, the calculated and experimental energies of the proton channels have been used in these refs). In the present work we use the spin-isospin part of the p-h interaction $F_{\sigma \tau}\left(x, x^{\prime}\right)=\left(\boldsymbol{\sigma} \boldsymbol{\sigma}^{\prime}\right)\left(\boldsymbol{\pi} \boldsymbol{\pi}^{\prime}\right) F_{\sigma \tau}\left(\mathbf{r}, \mathbf{r}^{\prime}\right)$ in the following forms:

$F_{\sigma \tau}\left(\mathbf{r}, \mathbf{r}^{\prime}\right)=F_{\sigma \tau} \delta\left(\mathbf{r}-\mathbf{r}^{\prime}\right)$

$F_{\sigma \tau}\left(\mathbf{r}, \mathbf{r}^{\prime}\right)=K_{\sigma \tau} f_{w s}(r) f_{w s}\left(r^{\prime}\right)$

The strength parameters in eqs. (2) $F_{\sigma \tau}=g^{\prime} \cdot 300 \mathrm{MeV} \cdot \mathrm{fm}^{3}, g^{\prime}=0.76$ and $K_{\sigma \tau}=0.11$ $\mathrm{MeV}$ are found so that the energy of the main maximum of the GT strength function calculated within the continuum-RPA would be equal to the experimental GTR energy. Notice in this connection that in the present work we use the determination of $F_{\sigma \tau}$ which is different from that has been used in the original edition of the monograph [19] and in [15, 16. This difference may not lead to some misunderstanding of our previous results.

Because the GT strength function calculated with the use of interactions (2) has no gross-structure in the GTR region, we evaluate the widths $\Gamma_{c}$ by the same way as in the case of the IAR. However, because the observable total width of the GTR is rather large 
$\left(\Gamma_{\text {tot }}=3.75 \mathrm{MeV}\right.$ [7]), we recalculate the widths $\Gamma_{c}$ to $\left\langle\Gamma_{c}\right\rangle$ using the proton penetrabilities averaged over the resonance. The calculated averaged widths $\left\langle\Gamma_{c}\right\rangle$ are systematically lager than nonaveraged ones (compare the results given in the seventh and eighth columns of Table 1). The comparison of two last columns of Table 1 shows that the use of the separable interaction $(2 \mathrm{~b})$ leads to the marked overestimation of the partial escape widths of the GTR.

Isoscalar monopole resonance. The partial neutron escape widths $\Gamma_{c}$ of the GMR in ${ }^{208} \mathrm{~Pb}$ have been calculated in [12, 15] with the use of the Landau-Migdal p-h interaction and calculated energies of the neutron decay channels. The calculated widths have been markedly overestimated as compared with the relevant experimental widths [4, 5]. In the present work we use the isoscalar part of the p-h interaction $F\left(x, x^{\prime}\right)=F\left(\mathbf{r}, \mathbf{r}^{\prime}\right)$ in two forms:

$F\left(\mathbf{r}, \mathbf{r}^{\prime}\right)=F(r) \delta\left(\mathbf{r}-\mathbf{r}^{\prime}\right)$

$F\left(\mathbf{r}, \mathbf{r}^{\prime}\right)=-K_{0}\left(d f_{w s}(r) / d r\right)\left(d f_{w s}\left(r^{\prime}\right) / d r^{\prime}\right)$

The radial-dependent strength $F(r)$ of the Landau-Migdal p-h interaction (3a) is a twoparametric function with a sharp change at the nuclear surface (see, e. g., [15]). The strength parameter $K_{0}=5.8 \mathrm{MeV} \cdot \mathrm{fm}^{2}$ in eq. (3b) is found so that the calculated energy of the GMR is consistent with experimental one. Because the monopole strength function calculated with the use of interactions (3) has no gross-structure in the GMR region, we evaluate the partial neutron escape widths $\left\langle\Gamma_{c}\right\rangle$ by the same way, which is used in the case of the GTR. Calculated results are given in Table 2 together with the relevant experimental data 4 , 5.

Table 2

Neutron escape widths (in keV) of GMR and GDR in ${ }^{208} \mathrm{~Pb}$

\begin{tabular}{|c|c|c|c|c|c|c|c|c|}
\hline \multirow{3}{*}{$\begin{array}{c}\text { final } \\
\text { state } \\
\text { of }{ }^{207} \mathrm{~Pb}\end{array}$} & \multicolumn{4}{|c|}{ GMR } & \multicolumn{4}{|c|}{ GDR } \\
\hline & \multicolumn{2}{|c|}{$\overline{\Gamma_{c}}(\exp )$} & \multicolumn{2}{|c|}{$\left\langle\Gamma_{c}\right\rangle($ calc $)$} & $\Gamma_{c}^{(\gamma)}$ & $\overline{\Gamma_{c}^{(n)}}$ & $\Gamma_{c}^{(\gamma)}$ & $\overline{\Gamma_{c}^{(n)}}$ \\
\hline & [5] & 41 & $\operatorname{int.}(3 a)$ & int.(3b) & int & 1a) & & (4) \\
\hline $1 / 2^{-}$ & 0 & $140 \pm 35$ & 76 & 49 & 42 & 75 & 1 & 23 \\
\hline $5 / 2^{-}$ & $<35$ & $70 \pm 15$ & 430 & 259 & 173 & 411 & 27 & 62 \\
\hline $3 / 2^{-}$ & $75 \pm 40$ & $50 \pm 10$ & 156 & 96 & 71 & 103 & 17 & 42 \\
\hline $13 / 2^{+}$ & $75 \pm 35$ & incl.in $3 / 2^{-}$ & 11 & 1.5 & 9 & 188 & 6 & 247 \\
\hline $7 / 2^{-}$ & $<140 \pm 30$ & $165 \pm 40$ & 323 & 169 & 7 & 7 & 31 & 38 \\
\hline $9 / 2^{-}$ & & & & & 8 & 11 & 8 & 11 \\
\hline$\Gamma_{t o t}^{\uparrow}$ & 325 & 425 & 996 & 574 & 310 & & 90 & \\
\hline
\end{tabular}

The use of the separable interaction (3b) somewhat decreases the calculated widths. Nevertheless, they are also overestimated as compared to the experimental widths. It can be expected that the use of the separable interaction as a superposition of surface and volume parts could allow us to describe the experimental widths adequately. These calculations are in progress now.

Giant dipole resonance. The GDR is an example of the giant resonance exhibiting some essential gross-structure within the continuum-RPA (see, e. g., [9, 15]). To describe the direct nucleon decay of such a GR it is necessary to calculate the cross sections $\sigma_{e N_{c}}$ 
and $\sigma_{e}$ of the reactions which are studied experimentally. An example of the calculation is given in [17], where the radiative channel has been considered as entrance one, so that $\sigma_{e N_{c}}$ and $\sigma_{e}$ are the cross sections of the partial $\left(\gamma N_{c}\right)$-reaction and photoabsorption, respectively. The satisfactory description of the experimental ${ }^{208} \mathrm{~Pb}\left(n \gamma_{0}\right)$-reaction cross section $\sigma_{n \gamma_{0}}^{e x p}$ in the GDR region has been obtained in [17] with the use of interaction (1a) and the experimental cross section of photoabsorption $\sigma_{a}^{e x p}$ [20. The branching ratio $b^{(\gamma)}=\sum_{c} \sigma_{\gamma n_{c}} / \sigma_{a} \simeq 10 \%$ calculated for the GDR energy is in qualitative agreement with the value $b^{\text {exp }} \simeq 3-5 \%$ deduced from the ${ }^{208} \mathrm{~Pb}\left({ }^{17} O,{ }^{17} \mathrm{O}^{\prime} n_{c}\right)$-reaction cross-sections [6].

In the present work we also use the separable $\mathrm{p}-\mathrm{h}$ interaction :

$F_{\tau}\left(\mathbf{r}, \mathbf{r}^{\prime}\right)=K_{1} \mathbf{r r}^{\prime}$

where the strength parameter $K_{1}=0.014 \mathrm{MeV} \cdot \mathrm{fm}^{-2}$ is found so that the calculated mean dws energy would be equal to the experimental GDR energy 20. The calculated cross section $\sigma_{n \gamma_{0}}$ is markedly underestimated in the GDR region (by a factor of 3) as compared to the relevant experimental value.

The calculations performed with the use of interactions (1a) and (4) show that the rootmean-squared dispersion of the dws energies is somewhat less than the GDR spreading width. For this reason one can roughly describe the cross sections $\sigma_{\gamma n_{c}}$ in the GDR region by means of the Breit-Wigner formulae in terms of some effective partial neutron widths of the GDR $\Gamma_{c}^{(\gamma)}$ [17]. However, due to the GDR gross-structure, these widths can be different, for example, from the effective partial neutron widths $\Gamma_{c}^{(n)}$ describing the GDR in the energy-averaged cross section of the elastic neutron-nucleus scattering [15. This statement is illustrated by the calculation results given in Table 2 .

4. The approach for description of the direct nucleon decay of the giant resonances with not-too-large excitation energy is briefly outlined. The approach is based on the exact version of the continuum-RPA and phenomenological consideration of the doorway-state coupling to many-particle configurations. The approach is applied to describe the direct nucleon decay of a number of GR in ${ }^{208} \mathrm{~Pb}$ parent nucleus. The comparison of calculation results with the relevant values deduced from experimental cross sections shows that the use of the Landau-Migdal p-h interaction is preferable for the description of the isovector giant resonances (IAR, GTR, GDR) as compared with the use of separable interactions. In the case of the GMR the situation is opposite. The main conclusion follows from the above consideration: the experimental and theoretical studies of the direct nucleon decay of giant resonances with not-too-large excitation energy allow one to get information on the particle-hole structure of these GR and on the particle-hole interaction in nuclei. For this reason continuation of these studies seems to be necessary.

5. The research described in this publication was made possible in part by Grant MQ2000 from the International Science Foundation (ISF), Grant MQ2300 from the ISF and Russian Government, and Grant 95-02-05917-a from Russian Found of Fundamental Researches (RFFR). Two authors (G.A.Ch. and M.H.U.) are grateful to the International Soros Science Education Program for support (respectively, Grants a73-f and 444p from the Open Society Institute, N.Y.). One of the authors (M.H.U.) is grateful to the RFFR for travel-grant 95-02-07892d. Two authors (S.E.M. and M.H.U.) are grateful to Prof. M.N.Harakeh as the Conference Chairperson for the local support. 


\section{References}

[1] S.Y.van der Werf et al., Phys.Lett. B216 (1989) 15

[2] O.A.Rumyantsev and M.H.Urin, Phys.Rev. C49 (1994) 537

[3] I.Bergqvist, D.M.Drake and D.K.McDaniels, Nucl.Phys. A191 (1972) 641

[4] A.Bracco et al., Phys.Rev.Lett. 60 (1988) 2603

[5] S.Brandenburg et al., Phys.Rev.C39 (1989) 2448

[6] A.van der Woude et al., Nucl.Phys. A569 (1994) 383c

[7] H.Akimine et al., Nucl.Phys.A569 (1994) 255c

[8] N. Van Giai et al., Phys.Lett. B233 (1989) 1

[9] N. Van Giai and Ch. Stoyanov, Phys.Lett. B252 (1990) 9

[10] T. Vertse et al., Phys.Lett. B264 (1991) 1

[11] G.Colo et al., Phys.Lett. B276 (1992) 279

[12] S.E.Muraviev and M.H.Urin, Phys.Lett. B280 (1992) 1

[13] E.Maglione, R.J.Liotta and T.Vertse, Phys.Lett. B298 (1993) 1

[14] G.Colo et al., Phys.Rev. C50 (1994) 1496

[15] S.E.Muraviev and M.H.Urin, Nucl.Phys. A572 (1994) 267

[16] S.E.Muraviev and M.H.Urin, Proc. IV Int.Conf.on Selected Topics in Nuclear Structure (Dubna,July 1994) p.232

[17] G.A.Chekomazov and M.H.Urin, Phys.Lett. B354 (1995) 7

[18] V.G.Guba and M.G.Urin, Nucl.Phys. A460 (1986) 222

[19] A.B.Migdal, Theory of finite Fermi-systems and applications to atomic nuclei (New York, Interscience, 1967)

[20] B.L.Berman and S.C.Fultz, Rev.Mod.Phys. 47 (1975) 713 